\title{
Tumores Estromales Extra-gastrointestinales (EGISTs): Revisión de 4 Casos con Análisis Inmunohistoquímico
}

\author{
Extra-gastrointestinal Stromal Tumors (EGISTs): \\ Review of Four Cases with Immunohistochemical Analysis
}

"Ismael Bernardo Fonseca; *Julio César Guerini; **Inés Strelzik;

***:Graciela Ghirardi; "Luis Gramática; *Rodolfo Esteban Avila \& "Luis Santos Spitale

FONSECA, I. B.; GUERINI, J. C.; STRELZIK, I.; GHIRARDI, G.; GRAMÁTICA, L.; AVILA, R. E. \& SPITALE, L. S. Tumores estromales extra-gastrointestinales (EGISTs): Revisión de 4 casos con análisis inmunohistoquímico. Int. J. Morphol., 28(4):991$998,2010$.

RESUMEN: Los tumores del estroma gastrointestinal o GISTs son neoplasias pertenecientes a los sarcomas de partes blandas y pueden localizarse en cualquier sitio del organismo. Los más frecuentes ocurren en el tracto gastrointestinal (del esófago al recto), de hecho, más del $50 \%$ se presentan a nivel gástrico. No obstante, se han descrito en otras regiones (mesenterio y omento, retroperitoneo, vesícula biliar, pared vesical y útero). Estos últimos de localización extragastrointestinal han sido publicados con el acrónimo EGIST y representan el $5 \%$ de todos los GISTs. Se presentan 4 casos de EGIST, describimos los hallazgos anatomopatológicos, los aspectos clínicos y el perfil inmunohistoquímico. Se estudiaron 32 casos de GISTs mediante análisis prospectivo y retrospectivo, de los archivos correspondientes al Servicio y II ${ }^{a}$ Cátedra de Patología de la Facultad de Ciencias Médicas, Hospitales Córdoba y Nuestra Sra. de la Misericordia, de Córdoba (Argentina), entre los años 1987 y 2008. De dicha serie, se extrajeron y analizaron cuatro casos (2 mesentéricos y 2 retroperitoneales). Todos los casos fueron pacientes de sexo femenino entre 42 y 72 años, que consultaron por dolor abdominal, dolor pélvico y pérdida de peso. El tamaño tumoral osciló entre 4 y $28 \mathrm{~cm}$. Todas las masas tumorales eran parcialmente quísticas, exhibiendo hemorragia y necrosis, en grado variable. Microscópicamente, se observaron los 2 patrones: fusocelular y epitelioide, de aspecto laxo, dispuestos en haces entrelazados con bajo índice mitótico. Panel inmunohistoquímico: Vimentina, CD34 y CD117: Positivos. Actina, cromogranina y S-100: Negativos. Ki 67: Positivo del 2 al $10 \%$. Diagnóstico: Tumor estromal extragastrointestinal de tipo borderline. La principal peculiaridad de los casos fue su localización. Los criterios diagnósticos de certeza deben considerar, en conjunto: los aspectos clínicos, histopatológicos convencionales y el perfil inmunohistoquímico.

PALABRAS CLAVE: GIST; c-kit; Tumores estromales extragastrointestinales; Células intersticiales de Cajal; Retroperitoneo; Mesenterio; Borderline.

\section{INTRODUCCIÓN}

Los tumores del estroma gastrointestinal o GISTs (del inglés gastrointestinal stromal tumors) pertenecen al grupo de los sarcomas de partes blandas (SPB) y como tales, pueden localizarse en cualquier otro sitio del organismo. Sin embargo, lo más frecuente es que dicha localización sea en el tracto gastrointestinal (desde esófago a recto) y, de hecho, más del $50 \%$ de los casos se presentan a nivel gástrico (Rosai, 2004). No obstante, se han descrito en otras localizaciones tales como mesenterio y omento (Suzuki et al., 2003), retroperitoneo (Reith et al., 2000), vesícula biliar
(Ortiz-Hidalgo et al., 2000), pared vesical (Lasota et al., 2000) y útero (Wingen et al., 2005). Estos últimos de localización extragastrointestinal han sido publicados con el acrónimo EGIST (del inglés Extra-gastrointestinal stromal tumor) y representan el 5\% de todos los GISTs (Yamamoto et al., 2004; Hou et al., 2003; Nakagawa et al., 2005).

La incidencia de los GISTs es baja, estimándose en 10-20 casos por millón de habitantes y año (Kindblom, 1998). La edad media de presentación se sitúa entre 50-70 años y

* Universidad Nacional de Córdoba, Facultad de Ciencias Médicas, II ${ }^{a}$ Cátedra de Patología, Argentina.

** Hospital Nuestra Señora de la Misericordia, Argentina.

${ }^{* * * *}$ Hospital Córdoba, Argentina. 
mayoritariamente en hombres (Rosai). Las manifestaciones clínicas dependen de su tamaño y localización, siendo las más frecuentes el dolor abdominal, la hemorragia digestiva y la masa abdominal, aunque muchos son asintomáticos (Roberts \& Eisenberg 2002).

El objetivo del presente trabajo es comunicar 4 casos de EGISTs, de localización mesentérica y retroperitoneal, describir los hallazgos anatomopatológicos, comentar aspectos clínicos y determinar su perfil inmunohistoquímico.

\section{MATERIAL Y MÉTODO}

Se estudiaron 32 casos de tumores estromales gastrointestinales en un análisis prospectivo y retrospectivo, de los archivos correspondientes al Servicio y II $^{\mathrm{a}}$ Cátedra de Patología de la Facultad de Ciencias Médicas, Hospital Córdoba y Hospital Nuestra Sra. de la Misericordia, Córdoba (Argentina), entre los años 1987 y 2008.

El material para estudio se fijó en una solución de formaldehído al $10 \%$ y luego se incluyó en parafina, realizando las tinciones habituales con H\&E y técnicas histoquímicas (PAS y tricrómico de Masson). De dicha serie se analizaron cuatro casos de localización extragastrointestinal (2 mesentéricos y 2 retroperitoneales).

Inmunohistoquímica: Se utilizó el sistema de alta sensibilidad Biotina - Estreptavidina - Peroxidasa - DAB (inmunodetector HRP/DAB, BIOSB) con el siguiente panel: Vimentina, CD34, c-kit (CD117), actina de músculo liso, S-100, desmina, AE1AE3, cromogranina y Ki 67.

Tratamiento estadístico de los resultados: Los resultados de los parámetros estudiados se analizaron con un modelo lineal de varianza y de normalidad. Luego se efectuó la comparación de las combinaciones posibles mediante datos categóricos. Por otra parte, los cambios anatomopatológicos observados se estudiaron a través del test de chi cuadrado para variables cualitativas. Para todos los casos, se estableció un nivel de significación de 0,05.

\section{RESULTADOS}

Caso Clínico 1: Mujer de 42 años, que consultó por dolor abdominal localizado en fosa iliaca izquierda. La laparotomía exploradora reveló en retroperitoneo una masa tumoral encapsulada.
Caso Clínico 2: Mujer de 50 años que consultó por dolor pélvico y pérdida de peso. Se realizó ecografía que confirmó una masa quística localizada en retroperitoneo.

Casos Clínicos 3 y 4: Mujer de 45 y 72 años, respectivamente, que consultaron por dolor abdominal, cuyas ecografías informaron masas tumorales voluminosas, parcialmente quísticas.

Protocolos N 1434/03, 2590/04 Hospital Córdoba.

Macroscopía: Formaciones ovoideas que miden $11 \mathrm{~cm} \mathrm{y} 4 \mathrm{~cm}$ respectivamente, recubiertas por una capsula translucida. Presentan superficie externa lobulada. Al corte son multinodulares con áreas mixoides y aspecto fasciculado (Fig. 1).

Protocolos No 41762 y 45321/08. Hospital Misericordia.

Macroscopía: Dos formaciones tumorales de 21 y $28 \mathrm{~cm}$ de diámetro cada una. $\mathrm{Al}$ corte parcialmente quísticas, con áreas de necrosis y de hemorragia.

Microscopía: Los preparados histológicos revelaron, en todos los casos, una proliferación fusocelular y epitelioide, de aspecto laxo, dispuesta en haces entrelazados con bajo índice mitótico (menos de 5 mitosis cada 50 campo de gran aumento) y sin atipia, en la que alternan dos patrones celulares, epitelioide y fusocelular (este último en forma predominante). Se identifican, además, áreas de patrón organoide, y otras mixoides (Figs. 2 y 3 ) hialinosis, focos de hemorragia y presencia de fibras esquenoides (Fig. 2). En los 2 casos de mayor tamaño tumoral se observaron extensas zonas necrohemorrágicas.

Panel inmunohistoquímico: Vimentina, CD34 (Fig. 4) y ckit (CD117) (Fig. 5), resultando positivos los 3 marcadores. Fueron negativos: actina de músculo liso, S-100, desmina, AE1AE3 y cromogranina. Ki 67 + (en un valor que osciló entre el 2 y el $10 \%$ ).

El diagnóstico en todos los casos estudiados (4) fue: Tumor estromal extragastrointestinal (EGIST) de tipo borderline (bajo grado de maligninidad).

\section{DISCUSIÓN}

En cuanto al origen de los GISTs, se postula que derivan de la transformación de las células intersticiales de Cajal (CIC) (Hirota et al., 1998; Kindblom et al.; Kitamura et al., 2003) o de un predecesor más inmaduro y pluripotencial (Miettinen, 1988). Histológicamente se distinguen tres cate- 


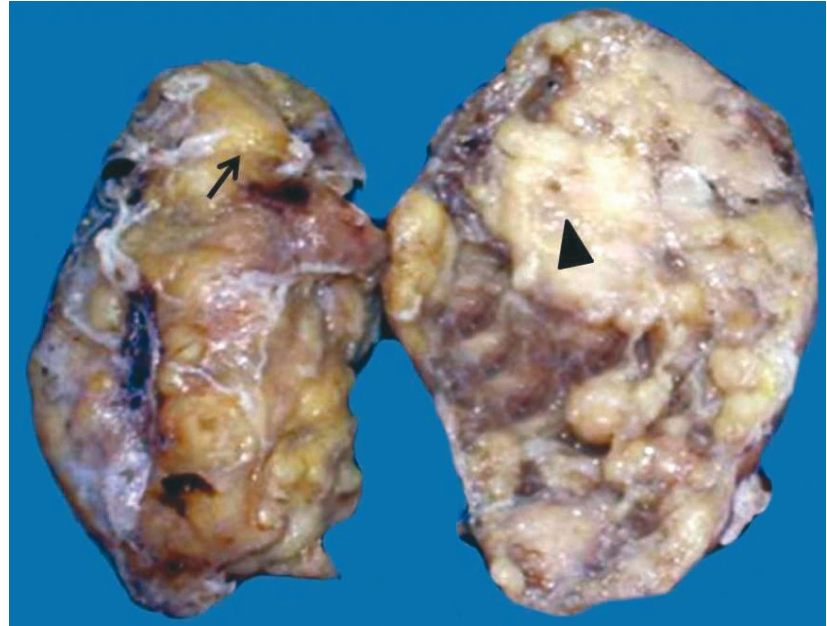

Fig. 1. EGISTs. Vista macroscópica de un tumor de localización retroperitoneal, con superficie externa lobulada (flecha). Al corte es sólido, blanquecino y multinodular (cabeza de flecha).

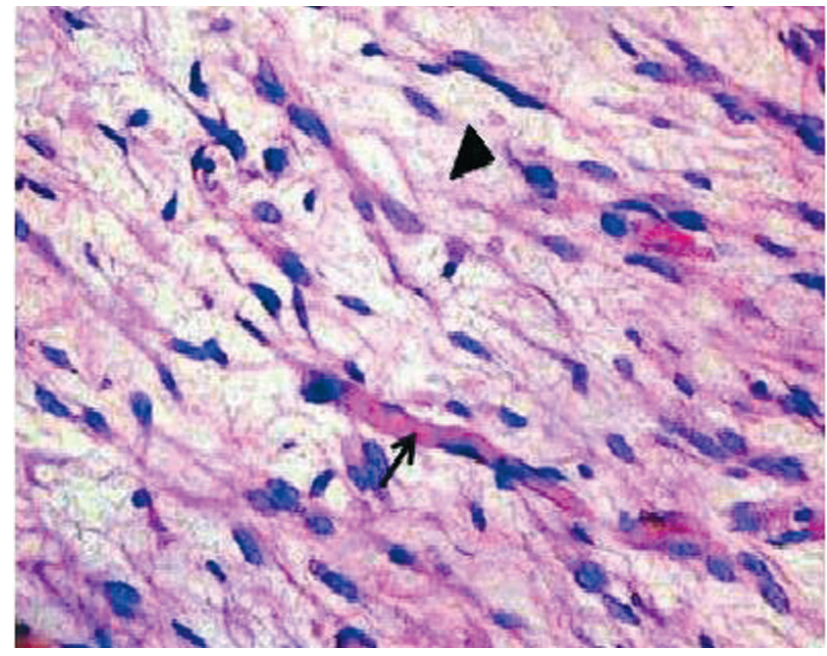

Fig. 2. Vista microscópica. EGIST con áreas mixoides, (punta de flecha) y presencia de fibras esquenoides (flecha). H\&E $40 \mathrm{X}$.

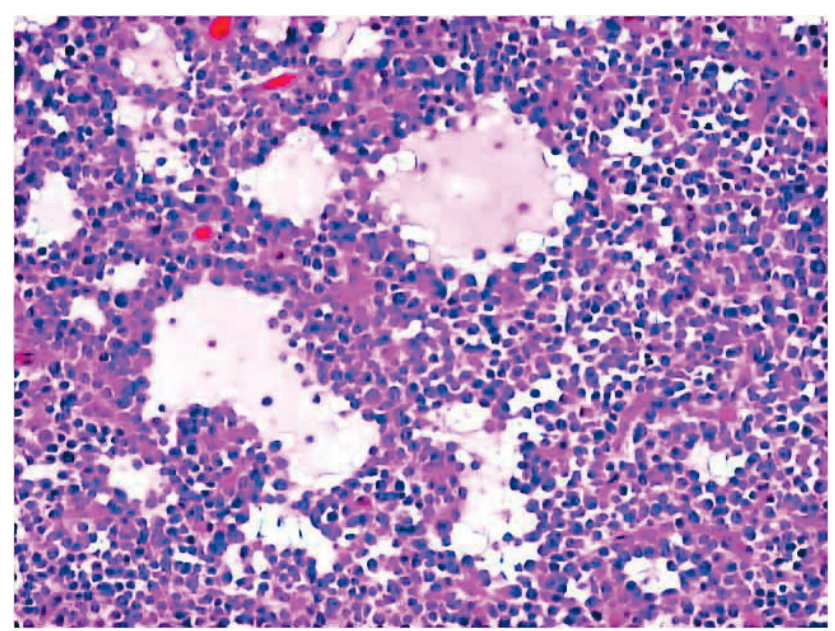

Fig. 3. Vista microscópica. EGIST en el que se observa una proliferación epiteloide con matriz mixoide H\&E 40X.

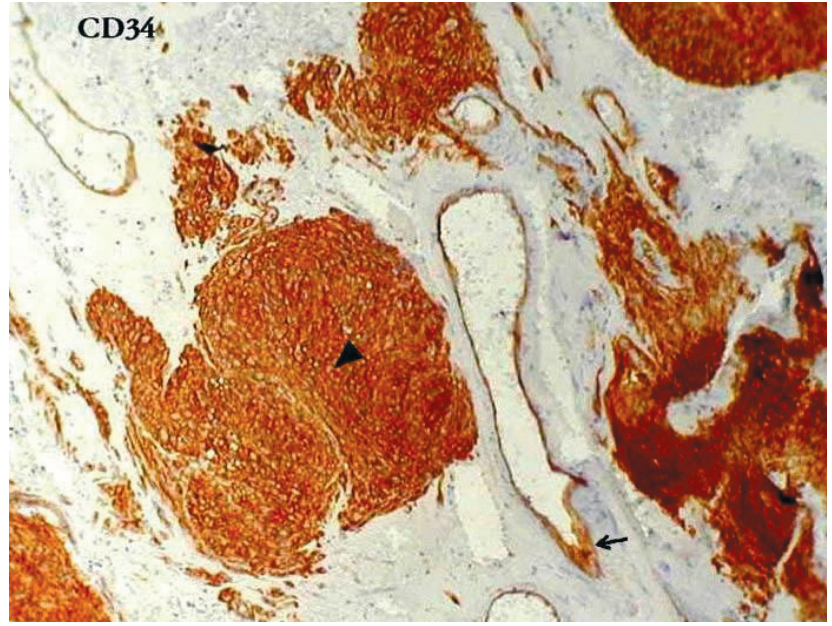

Fig. 4. Vista microscópica. EGIST. Se observa fuerte positividad de las células tumorales (punta de flecha). La tinción + en la pared vascular (flecha) sirve de control interno. Tinción inmunohistoquímica CD-34.100X.

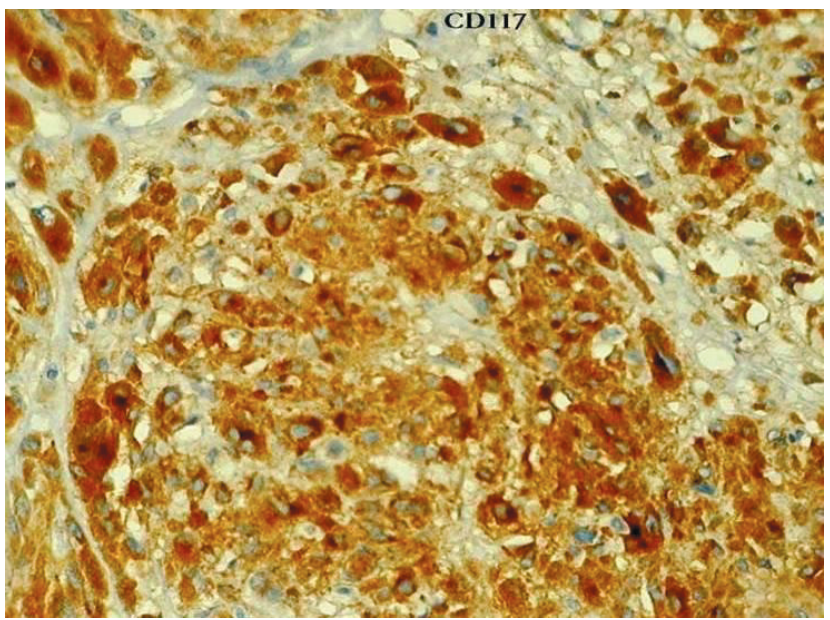

Fig. 5. Vista microscópica. EGIST. Patrón de tinción citoplasmático. Tinción inmunohistoquímica CD-117. 40X.

gorías: fusocelular (70\%), epitelioide (20\%) y mixta (5\%), sin que se sepa hasta el momento, que influencien el pronóstico (Miettinem \& Lasota, 2001).

La clave molecular de los GISTs es la proteína transmembrana KIT (CD117). Al contactar ciertos factores con su porción extracelular, se producen reacciones en cascada que activan su porción intracelular (tirosín-quinasa) con lo que la célula se multiplica con gran rapidez y pierde la capacidad de muerte programada o apoptosis (Pawson, 2002; Schlessinger, 1988).

Por otra parte, c-Kit es el gen que codifica dicha proteína. Se localiza en el brazo largo del cromosoma 4 y posee 21 exones. En 1999, se demostró que mutaciones de c-Kit (sobre todo en los exones 11 y 9) daban lugar a formas defec- 
tuosas de la proteína KIT y estas formas mutantes permanecían activadas permanentemente, incluso sin estímulos externos y escapando a los mecanismos de control capaces de "desconectar" la forma normal de la proteína (Hirota et al.; Rubin et al., 2001; Rubin et al., 2000).

Desde el punto de vista de la inmunohistoquímica el 60-70\% de los GISs expresan positividad para CD-34, un 30$40 \%$ son positivos para actina de músculo liso y un 5-10\% son positivos para el antígeno de la proteína S-100; la desmina es positiva en el 1-2\% de los casos. Sin embargo, ninguno de ellos es específico para diagnosticar GIST en ausencia de positividad para KIT. En 1998, Hirota et al. comunicaron la expresión de la proteína KIT, pudiendo ser detectada con el anticuerpo monoclonal CD-117. Con esta técnica, se diagnostican el 9495\% de los GIST. Heinrich et al., 2002, han encontrado mutaciones en el gen PDGFRa en casos de c-Kit negativos y añaden que ambas expresiones son mutuamente excluyentes, pero con consecuencias biológicas similares y sin poder concretar si en el momento actual estos hallazgos tendrían repercusiones pronósticas o terapeúticas. Por otra parte, hay GIST que son cKit negativos y tumores c-Kit positivos que no son GIST (Yamamoto et al.). Otro marcador inmunohistoquímico que podría ser útil en los c-Kit negativos, es la proteína quinasa $\mathrm{C}$ theta (PKC-theta) (Motegi et al., 2005).

En la Tabla I se resumen los criterios pronósticos propuestos por Fletcher (Fletcher, 2002).

El tratamiento del GIST resecable es la cirugía completa con bordes amplios y libres; sin embargo, a veces no es posible por el gran tamaño de la tumoración. En estos casos, así como en la enfermedad metastásica, el tratamiento es el mesilato de imatinib (Gleevec $\left.{ }^{\circledR}\right)$. En la actualidad se están llevando a cabo ensayos clínicos en adyuvancia en pacientes con factores de mal pronóstico que, sin duda, permitirán un mejor abordaje de la neoplasia.

El término tumor estromal gastrointestinal ha ganado amplia aceptación en la literatura, enfatizando su origen enigmático, dado que la mayoría de estas lesiones no mues- tra una diferenciación muscular o neuronal convincente (Appelman \& Helwig, 1976; Miettinen), revelando un perfil inmunofenotípico variable.

Kindblom et al. sostienen que el vocablo GISTs representa un término reservado, evasivo y no comprometido, mientras que otros autores creen que una significativa proporción de estas neoplasias representan tumores de músculo liso (Ma et al., 1993; Saul et al., 1987; Taniguchi et al., 1999; Tworek et al., 1997).

A fines de la década del 80, algunos estudios demostraron casos de GISTs con diferenciación neural, siendo introducido el término "tumor autonómico gastro-intestinal" (gastrointestinal autonomic nerve tumours - GANT), los cuales fueron previamente reconocidos por Herrera et al. (1989) como "plexo-sarcomas".

Walker \& Dvorak (1986) introdujeron el término "tumor del sistema nervioso autónomo gastrointestinal" (GANT) debido a determinados hallazgos al examen con microscopia electrónica, tales como, diferenciación axonal y presencia de gránulos electrodensos.

El descubrimiento de las mutaciones de hiperactivación en el protooncogén c-Kit por Hirota et al. fue muy importante en la génesis y clasificación de estos tumores y su tratamiento específico posterior.

Desde el punto de vista histológico, la situación es complicada y pueden existir errores de diagnóstico por cuanto algunos tumores muestran un patrón mioide o schwannoide, además de expresar arbitrariamente actina de músculo liso, desmina o el antígeno de la proteína S-100. En los casos que presentamos, la inmunohistoquímica fue negativa para actina de músculo liso.

Histológicamente, los tumores analizados en nuestra serie estaban constituidos por una población de células fusiformes, isomorfas sin atipias, que se disponían en fascículos entrecruzados y en empalizada. Tras la determinación

Tabla I. EGIST. Factores pronósticos.

\begin{tabular}{lcc}
\hline \multicolumn{3}{c}{ Factores Pronósticos en EGIST } \\
\hline Grado de riesgo & Tamaño & n de mitosis \\
\hline Muy bajo & $<2 \mathrm{~cm}$ & $<5$ X 50 CGA \\
Bajo & $2-5 \mathrm{~cm}$ & $<5 \times 50$ CGA \\
Intermedio & $<5 \mathrm{~cm}$ & $6-10 \times 50$ CGA \\
& $5-10 \mathrm{~cm}$ & $<5 \times 50$ CGA \\
Alto & $>5 \mathrm{~cm}$ & $>5 \times 50$ CGA \\
& $>10 \mathrm{~cm}$ & Cualquiera \\
& Cualquiera & $>10 \times 50$ CGA \\
\hline
\end{tabular}


de CD-117, se catalogaron de tumor estromal extragastrointestinal (2 mesentéricos y 2 retroperitoneales). Como factores de pronóstico adversos se encontraron el tamaño (el mayor de ellos medía $28 \mathrm{~cm}$ de diámetro) y las áreas de necrosis y de hemorragia.

Se han publicado trabajos donde se analiza la expresión Ki-67: si es $>10 \%$ existe mayor riesgo de recidiva o de metástasis, aunque quizá el índice sea sólo reflejo de la actividad mitótica (Rudolph et al., 1998; Seidal \& Edvardsson, 1999). En nuestros casos, el Ki-67 osciló del 2 al 10\% y como se ha mencionado, la actividad mitótica fue baja.

Otros criterios, como la expresión del factor de crecimiento endotelial vascular (VEGF) (Takahashi et al., 2003), la pérdida de expresión de CD 44 (Montgomery et al., 2004) y las alteraciones de p16 (Schneider-Stock et al., 2003) que se relacionan con peor pronóstico, no fueron analizadas en los casos aquí presentados.

Si sabemos en la actualidad que estos tumres estromales se pueden localizar en zonas extragastro-intestinales ¿por qué seguimos denominándolos "gastrointestinal stromal tumors o GIST? ¿en cuántas ocasiones han dejado de diagnosticarse por radicarse en esas zonas y, por tanto, no ser compatibles con el concepto anatómico? \& puede esto haber sido causa de una incidencia subestimada con sus consiguientes implicaciones terapéuticas?

En nuestra opinión, como la de otros autores (Contreras Ibañez et al., 2006), creemos que sería menos confuso el término de "tumores estromales con fenotipo de células de Cajal", con lo que se rendiría honor a su trabajo.

Las características particulares que exhiben los tumores estromales incluyen un abanico de conceptos sobre histogénesis, morfología con técnicas de rutina, inmunorreación, conducta clínica, pronóstico, alteraciones genéticas puntuales, terapéutica y otras que se investigan en la actualidad y que, sin lugar a dudas, serán de gran importancia en los próximos años.

Los EGISTs muestran expresión de c-Kit y apariencia histopatológica similar a los GISTs. Los tumores GIST, constituyen uno de los mas claros ejemplos del manejo multidisciplinario en interacción ordenada, en el cual deben participar: el patólogo, como determinante diagnóstico que intenta establecer los parámetros morfológicos de valor pronóstico, el clínico que establece la localización y extensión de la lesión, el cirujano, quien con su juicio crítico determina si la lesión es resecable, procurando reducir al mínimo la morbimortalidad, y el oncólogo, que ofrece la terapéutica previa o posterior a la resección quirúrgica.
La principal peculiaridad de los casos que presentamos es su localización. Los EGIST son tumores muy poco frecuentes (un 5\% del total de GIST) habiéndose publicado casos aislados y dos series en la literatura (Reith et al.; Miettinen et al., 1999). Como ocurre con otras neoplasias de partes blandas, los EGIST probablemente se originan en células muy inmaduras pluripotenciales que, en este caso, seguirían una línea de diferenciación hacia células c-Kit+ / tipo célula intersticial de Cajal. Se presentan en adultos, en epiplón o mesenterio y, menos frecuentemente, retroperitoneo (Reith et al.; Miettinen et al.). Generalmente, tienen gran tamaño ( $>10 \mathrm{~cm}$ de diámetro) cuando se diagnostican y con frecuencia (50\%) siguen un curso agresivo con recidivas o metástasis (Reith et al.; Miettinen et al.). Se han sugerido como criterios predictivos de evolución: el grado de celularidad, el índice mitótico y la existencia de necrosis (Miettinen et al.). Hay que tener en cuenta, sin embargo, que la positividad para c-Kit en una neoplasia mesenquimal no equivale sin más a un diagnóstico de EGIST. Cada vez se comunican más tumores de partes blandas que muestran, al menos en algún caso, expresión de c-Kit (Sabah et al., 2003). El hallazgo de estos casos se ve favorecido por la demanda creciente de los oncólogos para que se realice detección inmunohistoquímica de c-Kit en determinados pacientes para intentar un posible tratamiento con Gleevec ® (a pesar que sólo se ha demostrado su eficacia en los GIST). Por otra parte, se empiezan a describir EGIST en localizaciones nuevas y muy raras, como por ejemplo vejiga urinaria (Krokowski et al., 2003).

En conclusión, para un diagnóstico seguro de GIST / EGIST se deben considerar en conjunto: clínica, histología y un panel completo de inmunohistoquímica. Cabe recordar además, las diferencias que se han observado en los resultados de la técnica, dependiendo de la sensibilidad de los diferentes anticuerpos comercializados para c-Kit y de que se utilice desenmascaramiento antigénico con calor (Lonardo et al., 2003).

FONSECA, I. B.; GUERINI，J. C.; STRELZIK, I.; GHIRARDI, G.; GRAMÁTICA, L.; AVILA, R. E. \& SPITALE, L. S. Extra-gastrointestinal stromal tumors (EGISTs): Review of four cases with immunohistochemical analysis. Int. J. Morphol., 28(4):991-998, 2010.

SUMMARY: Gastrointestinal stromal tumors or GISTs are neoplasms belonging to the soft tissue sarcomas and can be located anywhere in the body. They most frequently occur in the gastrointestinal tract (esophagus to rectum), in fact, over $50 \%$ occur at the gastric level. However, they have also been described elsewhere (mesentery and omentum, retroperitoneum, gall bladder, bladder wall and uterus). Extragastrointestinal latter location have 
been published by the acronym EGIST (representing $5 \%$ of all GISTs. We report four cases of EGIST ( 2 of mesenteric and retroperitoneal location) describe the histopathological, clinical aspects and immunohistochemical profile. We studied 32 cases of GISTs in a prospective and retrospective analysis of files for the Service and IInd Chair of Pathology (Emergency Municipal Hospital), Hospital Córdoba, and Our Lady of Mercy, Cordoba (Argentina), between 1987 and 2008. In that series were extracted and analyzed four cases (two retroperitoneal and two mesenteric). All cases were female patients whose ages ranged from 42 to 72 years who consulted for abdominal pain, pelvic pain and weight loss. Tumor size ranged from 4 to $28 \mathrm{~cm}$. All were partially cystic mass, showing hemorrhage and necrosis, in varying degrees. Microscopic patterns were observed 2 spindle and epithelioid-looking lax, arranged in bundles intertwined with low mitotic index. Immunohistochemistry panel: Vimentin, CD34 and CD117: Positive. Actin, chromogranin and S-100: Negative. Ki 67: + 2 to 10\%. Diagnosis: extragastrointestinal stromal tumor, borderline. Complete histopathological and immunohistochemical panels should be considered for a definite diagnosis. The main peculiarity of the present cases is their location. The EGIST are rare (5\% of GIST).

KEY WORDS: GIST; c-Kit; Extragastrointestinal stromal tumors; Interstitial cells of Cajal; Retroperitoneum; Mesentery; Borderline.

\section{REFERENCIAS BIBLIOGRÁFICAS}

Appelman, H. D. \& Helwig, E. B. Gastric epithelioid leiomyoma and leiomyosarcoma (leiomyoblastoma). Cancer, 38:708-28, 1976.

Contreras Ibañez, J. A.; Atienza Cuevas, L.; Pérez Requena, J.; Beltrán Ruiz-Henestrosa, M. \& Baena Cañada, J. M. GIST: A propósito de un término confuso y presentación de un caso de tumor del estroma extragastrointestinal. Oncologia, 29(6):30-4, 2006.

Fletcher, C. D. Clinicopathologic correlations in gastrointestinal stromal tumors. Hum. Pathol., 33(5):455, 2002.

Heinrich, M. C.; Corless, C. L.; Duensing, A.; McGreevey, L.; Chen, C. J.; Joseph, N.; Singer, S.; Griffith, D. J.; Haley, A.; Town, A.; Demetri, G. D.; Fletcher, C. D. \& Fletcher, J. A. PDGFRA activating mutations in gastrointestinal stromal tumors. Science, 299(5607):70810, 2003.

Herrera, G. A.; Cerezo, L.; Jones, J. E.; Sack, J.; Grizzle, W. E.; Pollack, W. J. \& Lott, R. L. Gastrointestinal autonomic nerve tumors. 'Plexosarcomas'. Arch. Pathol. Lab. Med., 113:846-53, 1989.
Hirota, S.; Isozaki, K.; Moriyama, Y.; Hashimoto, K.; Nishida, T.; Ishiguro, S.; Kawano, K.; Hanada, M.; Kurata, A.; Takeda, M.; Muhammad Tunio, G.; Matsuzawa, Y.; Kanakura, Y.; Shinomura, Y. \& Kitamura, Y. Gain-of-function mutations of c-kit in human gastrointestinal stromal tumors. Science, 279(5350):577-80, 1998.

Hou, Y. Y.; Sun, M. H.; Wei, Y. K.; Tan, Y. S.; Lu, X. Y.; Wang, J.; Zhu, X. Z. \& Zheng, A. H. Clinicopathological, immunohistochemical and molecular genetic study of intra-abdomen extragastrointestinal stromal tumors. Zhonghua Bing Li Xue Za Zhi, 32(5):422-6, 2003.

Kindblom, L. G.; Remotti, H. E.; Aldenborg, F. \& MeisKindblom, J. M. Gastrointestinal pacemaker cell tumor (GIPACT): gastrointestinal stromal tumors show phenotypic characteristics of the interstitial cells of Cajal. Am. J. Pathol., 152(5):1259-69, 1998.

Kitamura, Y.; Hirota, S. \& Nishida, T. Gastrointestinal stromal tumors (GIST): A model for molecule-based diagnosis and treatment of solid tumors. Cancer Sci., 94(4):315-20, 2003.

Krokowski, M.; Jocham, D.; Choi, H.; Feller, A. C. \& Horny, H. P. Malignant extragastrointestinal stromal tumor of bladder. J. Urol., 169:1790-1, 2003.

Lasota, J.; Wozniak, A.; Sarlomo-Rikala, M.; Rys, J.; Kordek, R.; Nassar, A.; Sobin, L. H. \& Miettinen, M. Mutations in exons 9 and 13 of KIT gene are rare events in gastrointestinal stromal tumors. A study of 200 cases. Am. J. Pathol., 157(4):1091-5, 2000.

Lonardo, F.; Pass, H. I. \& Lucas, D. R. Immunohistochemistry frequently detects c-Kit expression in pulmonary small cell carcinoma and may help select clinical subsets for a novel form of chemotherapy. Appl. Immunohistochem. Mol. Morphol., 11(1):51-5, 2003.

Ma, C. K.; Amin, M. B.; Kintanar, E.; Linden, M. D. \& Zarbo, R. J. Immunohistologic characterization of gastrointes-tinal stromal tumors: a study of 82 cases compared with 11 cases of leiomyomas. Mod. Pathol., 6:139-44, 1993.

Miettinen, M. Gastrointestinal stromal tumors. An immunohistochemical study of cellular differentiation. Am. J. Clin. Pathol., 89(5):601-10, 1988. 
Miettinem, M. \& Lasota, J. Gastrointestinal stromal tumors-definition, clinical, histological, immunohistochemical, and molecular genetic features and differential diagnosis. Virchows Arch., 438:1-12, 2001.

Miettinen, M.; Monihan, J. M.; Sarlomo-Rikala, M.; Kovatich, A. J.; Carr, N. J.; Emory, T. S. \& Sobin, L. H. Gastrointestinal stromal tumors / smooth muscle tumors (GISTs) primary in the omentum and mesentery. Clinicopathologic and immunohistochemical study of 26 cases. Am. J. Surg. Pathol., 23:1109-18, 1999.

Montgomery, E.; Abraham, S. C.; Fisher, C.; Deasel, M. R.; Amr, S. S.; Sheikh, S. S.; House, M.; Lilliemoe, K.; Choti, M.; Brock, M.; Ephron, D. T.; Zahuruk, M. \& Chadburn, A. CD44 loss in gastric stromal tumors as a prognostic marker. Am. J. Surg. Pathol., 28:168$77,2004$.

Motegi, A.; Sakurai, S.; Nakayama, H.; Sano, T.; Oyama, T. \& Nakajima, T. PKC-theta, a novel immunohistochemical marker for gastrointestinal stromal tumors (GIST), especially useful for identifying KIT-negative tumors. Pathol Int., 55(3):106-12, 2005.

Nakagawa, M.; Akasaka, Y.; Kanai, T.; Takabayashi, T. \& Miyazawa, N. Clinicopathological and immunohistochemical features of extragastrointestinal stromal tumors: report of two cases. Surg. Today, 35(4):336-40, 2005.

Ortiz-Hidalgo, C.; de Leon Bojorge, B. \& AlboresSaavedra, J. Stromal tumor of the gallbladder with phenotype of interstitial cells of Cajal: A previously unrecognized neoplasm. Am. J. Surg. Pathol., 24(10):1420-3, 2000.

Pawson, T. Regulation and targets of receptor tyrosine kinases. Eur. J. Cancer, 38(5):3-10, 2002.

Reith, J. D.; Goldblum, J. R.; Lyles, R. H. \& Weiss, S. W. Extragastrointestinal (soft tissue) stromal tumors: An analysis of 48 cases with emphasis on histologic predictors of outcome. Mod. Pathol., 13(5):577-85, 2000.

Roberts, P. J. \& Eisenberg, B. Clinical presentation of gastrointestinal stromal tumors and treatment of operable disease. Eur. J. Cancer, 38(5):S37-8, 2002.

Rosai, J. Controversies in surgical pathology. Int. J. Surg. Pathol., 12(4):299-300, 2004.
Rubin, B. P.; Fletcher, J. A. \& Fletcher, C. D. Molecular insights into the histogenesis and pathogenesis of gastrointestinal stromal tumors. Int. J. Surg. Pathol., 8(1):5-10, 2000.

Rubin, B. P.; Singer, S.; Tsao, C.; Duensing, A.; Lux, M. L.; Ruiz, R.; Hibbard, M. K.; Chen, C. J.; Xiao, S.; Tuveson, D. A.; Demetri, G. D.; Fletcher, C. D. \& Fletcher, J. A. KIT activation is a ubiquitous feature of gastrointestinal stromal tumors. Cancer Res., 61(22):8118-21, 2001.

Rudolph, P.; Peters, J.; Lorenz, D.; Schmidt, D. \& Parwaresch, R. Correlation between mitotic and Ki67 labeling indices in paraffin-embedded carcinoma specimens. Hum. Pathol., 29(11):1216-22, 1998.

Sabah, M.; Leader, M. \& Kay, E. The problem with kit: Clinical implications and practical difficulties with CD117 immunostaining. Appl. Immunohistochem. Mol. Morphol., 11:56-61, 2003.

Saul, S. H.; Rast, M. L. \& Brooks, J. J. The immunohistochemistry of gastrointestinal stromal tumors. Evidence supporting an origin from smooth muscle. Am. J. Surg. Pathol., 11:464-73, 1987.

Schlessinger, J. Signal transduction by allosteric receptor oligomerization. Trends Biochem. Sci., 13(11):443-7, 1988.

Schneider-Stock, R.; Boltze, C.; Lasota, J.; Miettinen, M.; Peters, B.; Pross, M.; Roessner, A. \& Günther, T. High prognostic value of p16 INK4 alterations in gastrointestinal tumors. J. Clin. Oncol., 21:1688-97, 2003.

Seidal, T. \& Edvardsson, H. Expression of c-kit (CD117) and $\mathrm{Ki} 67$ provides information about the possible cell of origin and clinical course of gastrointestinal stromal tumours. Histopathology, 34(5):416-24, 1999.

Suzuki, K.; Kaneko, G.; Kubota, K.; Horigome, N.; Hikita, H.; Senga, O.; Miyakawa, M.; Shimojo, H.; Uehara, T. \& Itoh, N. Malignant tumor, of the gastrointestinal stromal tumor type, in the greater omentum. $J$. Gastroenterol., 38(10):985-8, 2003.

Takahashi, R.; Tanaka, S.; Kitadai, Y.; Sumii, M.; Yoshihara, M.; Haruma, K. \& Chayama, K. Expression of vascular endothelial growth factor and angiogenesis in gastrointestinal stromal tumor of the stomach. Oncology, 64:266-74, 2003. 
FONSECA, I. B.; GUERINI, J. C.; STRELZIK, I.; GHIRARDI, G.; GRAMÁTICA, L.; AVILA, R. E. \& SPITALE, L. S. Tumores Estromales Extra- gastrointestinales (EGISTs): Revisión de 4 casos con análisis inmunohistoquímico. Int. J. Morphol., 28(4):991-998, 2010.

Taniguchi, M.; Nishida, T.; Hirota, S.; Isozaki, K.; Ito, T.; Nomura, T.; Matsuda, H. \& Kitamura, Y. Effect of c-kit mutation on prognosis of gastrointestinal stromal tumors. Cancer Res., 59:4297-300, 1999.

Tworek, J. A.; Appelman, H. D.; Singleton, T. P. \& Greenson, J. K. Stromal tumors of the jejunum and ileum. Mod. Pathol., 10:200-9, 1997.

Walker, P. \& Dvorak, A. M. Gastrointestinal autonomic nerve (GAN) tumor. Ultrastructural evidence for a newly recognized entity. Arch. Pathol. Lab. Med., 110:309-16, 1986.

Wingen, C. B.; Pauwels, P. A.; Debiec-Rychter, M.; van Gemert, W. G. \& Vos, M. C. Uterine gastrointestinal stromal tumour (GIST). Gynecol. Oncol., 97(3):970-2, 2005.

Yamamoto, H.; Oda, Y.; Kawaguchi, K.; Nakamura, N.; Takahira, T.; Tamiya, S.; Saito, T.; Oshiro, Y.; Ohta, M.; Yao, T. \& Tsuneyoshi, M. c-kit and PDGFRA mutations in extragastrointestinal stromal tumor (gastrointestinal stromal tumor of the soft tissue). Am. J. Surg. Pathol., 28(4):479-88, 2004.
Dirección para correspondencia:

Ismael Bernardo Fonseca

Rodriguez Peña 285

CEP: 5000

Hospital Universitario de Maternidad y Neonatología

Facultad de Ciencias Médicas

Universidad Nacional de Córdoba

Córdoba

ARGENTINA

Email: isma_fonseca@yahoo.com.ar

Recibido : 27-06-2010

Aceptado: 11-09-2010 